\title{
Application of analytical hierarchy process for Structural Health Monitoring and Prioritizing of Concrete Bridges in Iran
}

\author{
Saeid Darban ${ }^{1}$, Hosein Ghasemzadeh Tehrani ${ }^{2}$, Nader Karballaeezadeh ${ }^{2, *}$ and Amir Mosavi ${ }^{3, *}$ \\ 1 Department of Engineering, Azad University of Shahrood, Shahrood P.O.B. 3619943189, Iran; Sae- \\ id.d1366@yahoo.com (S.D.) \\ 2 Faculty of Civil Engineering, Shahrood University of Technology, Shahrood P.O.B. 3619995161, Iran; \\ H_ghasemzadeh@shahroodut.ac.ir (H.G.T.); N.karballaeezadeh@shahroodut.ac.ir (N.K.) \\ 3 Institute of Automation, Kalman Kando Faculty of Electrical Engineering, Obuda University, Budapest 1034, \\ Hungary; Amir.mosavi@nik.uni-obuda.hu (A.M.) \\ * Correspondence: N.karballaeezadeh@shahroodut.ac.ir, Amir.mosavi@nik.uni-obuda.hu;
}

\begin{abstract}
This paper proposes a method for monitoring the structural health of concrete bridges in Iran. In this method, the bridge condition index (BCI) of bridges is determined by the analytical hierarchy process. BCI constitutes eight indices that are scored based on the experts' views, including structural, hydrology and climate, safety, load impact, geotechnical and seismicity, strategic importance, facilities, and traffic and pavement. Experts' views were analyzed by Expert Choice software, and the relative importance (weight) of indices were determined using the analytical hierarchy process (AHP). Then, the gave scores of experts were assigned to indices for various conditions. Bridge inspectors can examine the bridge, determine the scores of indices, and compute BCI. Higher values of BCI indicate better conditions. Therefore, bridges with lower BCI take priority in maintenance activities. Five bridges in Iran, Semnan province, were selected as the case studies, and BCI calculation of these bridges was conducted.
\end{abstract}

Keywords: transportation infrastructure, concrete bridges, structural health monitoring, bridge condition index, analytical hierarchy process, prioritizing

\section{Introduction}

The quality of transportation systems directly affects the lives of urban residents. A large portion of the national resources of each country is invested in this area. As one of the most important parts of transportation systems, bridges have a critical role in urban development [1-6]. The bridge conditions in the transportation networks are so important that the costs incurred by out of service bridges are exorbitant. Therefore, bridges condition evaluation has crucial importance for the proper maintenance and management of transportation infrastructures. Another important factor that affects the maintenance process of infrastructures is budget constraints. Consequently, further attention should be paid to the development of a bridge management system (BMS) $[7,8]$. The first step in the BMS is to prepare a technical profile for all bridges in the network. This profile contains technical information such as the name of a bridge, its location, construction method, etc. It is, in fact, the starting point of BMS. The next step in BMS is assessment, including structural and seismic assessment, hydrological assessment, facility evaluation, safety assessment and pavement and traffic evaluation [9]. Bridge inspection methods are divided into four general categories [10,11]: 1. Visual assessment, 2. Evaluation by non-destructive tests, 3 . Sampling and destructive tests, and 4 . Health assessment. Another major step of BMS is bridge maintenance. The maintenance involves a variety of operations that continuously ensure the safety and serviceability of a bridge over its lifetime. The prioritization of bridge maintenance, including repairs or reinforcement, is the cornerstone of the BMS $[9,12]$. Traditionally, the prioritization of bridge maintenance projects was carried out based on engineer's assessment for small-sized bridges and for 
large and old bridge networks, it was conducted in accordance with concepts and principles of optimization in project budget allocation. Today, the bridge condition index (BCI) is used for this purpose. BCI is a good benchmark for prioritizing BMS [13].

The service life of a bridge is divided into four different phases [14]:

- Design and construction

- $\quad$ Start of damages (early damage stages)

- The spread of damages

- The expansion of damages

Under the famous Law of Five, each dollar spent on the first phase will equal $\$ 5$ in the second phase, $\$ 25$ in the third phase, and \$125 in the fourth phase [14]. According to this law, any miscalculated decisions about maintenance, repair and rehabilitation (MR\&R) in bridges would incur surplus costs. With this in mind, there is a need for decision support system (DSS). DDS aims at improving the bridge network condition and allocating budget appropriately [15]. Most of BMSs are founded upon processes that optimize the cost of a lifecycle and tend to overlook factors such as environmental impacts and social impacts. This gives rise to a number of problems, especially when the existing financial resources are higher or lower than the cost of computational life cycle [16].

In this paper, the main goal is to present an applicable method for determining the condition index of the concrete bridges in Iran. For this purpose, firstly, eight critical indices were selected. These indices consist of structure, hydrology, climate and hydrology, safety, load impact, geotechnical and seismicity, strategic importance, facilities, and traffic and pavement. Each index comprises a number of sub-indices. In the next step, a series of questionnaires were developed, which contained questions about the relative importance of the indices and their sub-indices. These questionaries were distributed among bridge experts. Experts' views and feedbacks were analyzed by Expert Choice software and the relative importance (weight) of all indexes and sub-indexes was determined using the analytical hierarchy process (AHP). In the end, bridge experts filled another questionnaire related to the condition score of each sub-index. The score of each index is the sum of condition scores assigned to its sub-indices. Finally, BCI is calculated as the sum of weighted scores assigned to indices. $\mathrm{BCI}$ is a value between 0 and 100, with higher values indicating a better bridge condition. Therefore, bridges with lower $\mathrm{BCI}$ take priority in terms of repair and maintenance. To test the proposed method in practice, five bridges in Semnan province, in Iran, were inspected and their BCI was determined to prioritize bridges in terms of maintenance requirements. This study is innovative because no comprehensive method has been proposed to evaluate and prioritize in-service bridges in Iran. Therefore, the proposed method helps Iranian engineers to evaluate and especially prioritize bridge maintenance operations more effectively.

This paper is organized as follows: Next section presents a literature review of BCI. Section "Method" introduces the study methodology, which is further divided into three general sub-sections entitled BCI, AHP, and sub-indices of BCI. The results are presented and discussed in section "Results and Discussion." The final section offers a summary of results and conclusions.

\section{Background}

The proper maintenance and management of bridges need the evaluation of safety and Lifetime conditions. In recent years, there is an increasing number of studies on the $\mathrm{BMS}$ and $\mathrm{BCI}$. This section presents a range of the most important methods used for determining BCI around the world.

Sanjay and Kumar developed a bridge health index (BHI) using AHP. They divided elements of the bridge into seven categories, including approaches, substructure, waterway/channel, foundations, superstructure, appurtenances/auxiliary works, and bearings. Then, they drafted a questionnaire and distributed it among engineers and experts. The results of the questionnaire were incorporated in determining the relative importance and weight of diverse elements. They also considered a numerical value for 
each type of damage. The condition of various elements of a bridge is assessed by visual inspection. Finally, BHI was developed by summing the score of all bridge elements [17]. In the Figure 1, the decision tree of this research is presented.

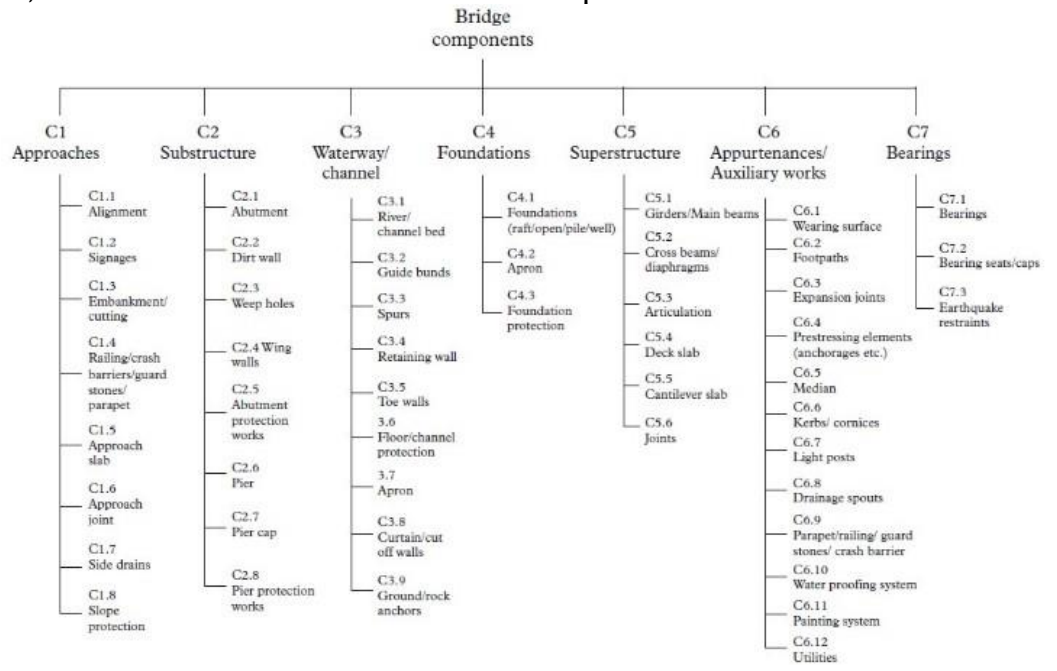

Figure 1. Decision tree in Sanjay and Kumar research.

In China, there are two main indexes for assessing bridge conditions. The Ministry of Transport of the People's Republic of China uses Dr index to assess the conditions of a bridge [18]:

$$
\mathrm{D}_{\mathrm{r}}=\mathrm{BDCI} \times \mathrm{W}_{\mathrm{D}}+\mathrm{SPCI} \times \mathrm{W}_{\mathrm{SP}}+\mathrm{SBCI} \times \mathrm{W}_{\mathrm{SB}}
$$

where $\mathrm{D}_{\mathrm{r}}$ is bridge condition rating, $\mathrm{BDCI}$ is bridge deck condition index, SPCI is bridge superstructure condition index, $\mathrm{SBCI}$ is bridge substructure condition index, $\mathrm{W}_{\mathrm{D}}, \mathrm{W}_{\mathrm{SP}}$, and $\mathrm{W}_{\mathrm{SB}}$ are the weight of BDCI, SPCI, and SBCI indicators, respectively. On the other hand, the Ministry of Housing and Urban-Rural Development of China has also provided a definition identical to Eq. 1 for assessing bridge conditions [19]:

$$
\mathrm{BCI}=\mathrm{BCI}_{\mathrm{d}} \times \omega_{\mathrm{d}}+\mathrm{BCI}_{\mathrm{sp}} \times \omega_{\mathrm{sp}}+\mathrm{BCI}_{\mathrm{sb}} \times \omega_{\mathrm{sb}},
$$

where $\mathrm{BCI}$ is bridge conditions index, $\mathrm{BCI}$ is bridge deck condition index, $\mathrm{BCI}_{\mathrm{sp}}$ is bridge superstructure condition index, $\mathrm{BCI}_{\mathrm{sb}}$ is bridge substructure condition index and $\omega-$ the weight of a bridge element. Table 1 presents the assessment approach based on $D_{r}$ and BCI.

Table 1. Assessment of bridge condition in China.

\begin{tabular}{ccc}
\hline $\mathrm{D}_{\mathrm{r}}$ & BCI & condition \\
\hline $95 \leq \mathrm{D}_{\mathrm{r}}<100$ & $90 \leq \mathrm{BCI}$ & Perfect \\
$80 \leq \mathrm{D}_{\mathrm{r}}<95$ & $80 \leq \mathrm{BCI}<90$ & Good (minor damage) \\
$60 \leq \mathrm{D}_{\mathrm{r}}<80$ & $66 \leq \mathrm{BCI}<80$ & Pass (mediate damage) \\
$40 \leq \mathrm{D}_{\mathrm{r}}<60$ & $50 \leq \mathrm{BCI}<66$ & Unqualified (great damage) \\
$0<\mathrm{D}_{\mathrm{r}}<40$ & $\mathrm{BCI}<50$ & Dangerous \\
\hline
\end{tabular}

In Japan, no specific formula or equation is used to evaluate the condition of a bridge. For each bridge, first one of status shown in Table 2 is assigned to each element based on the assessment of the bridge inspector, and then the bridge general conditions are described in accordance with Table 3 [20].

Table 2. Assessment of maintenance urgency for bridge element in Japan.

\begin{tabular}{cc}
\hline Rate & Condition \\
\hline A & No repair needed \\
\hline
\end{tabular}




\begin{tabular}{cc}
\hline B & No immediate repairs needed \\
C1 & Immediate repairs needed from standpoint of preventative maintenance \\
C2 & Immediate repairs needed from standpoint of structural safety \\
E1 & Immediate actions needed from standpoint of structural safety \\
E2 & Immediate actions needed in tandem with other factors \\
M & Repairs needed during regular maintenance work \\
S1 & In-depth investigations needed \\
S2 & Follow-up investigations needed \\
\hline
\end{tabular}

Table 3. Bridge soundness in Japan.

\begin{tabular}{ccc}
\hline State & Condition & Description \\
\hline 1 & Good & No problems in bridge's functions \\
2 & $\begin{array}{c}\text { Preventative } \\
\text { maintenance }\end{array}$ & No problems in bridge's functions but maintenance required from \\
standpoint of preventive maintenance
\end{tabular}

In Korea, a damage index (DI) is used to assess bridge conditions. It is the normalized index obtained from the evaluation of all bridge elements. The DI index is shown in Eq. (3) [21]:

$$
\begin{gathered}
\mathrm{DI}=\sum\left(\mathrm{CR}_{\mathrm{i}} \times \mathrm{WF}_{\mathrm{i}}\right) / 100, \\
\sum\left(\mathrm{WF}_{\mathrm{i}}\right)=100,
\end{gathered}
$$

where DI is damage index, $\mathrm{CR}_{\mathrm{i}}$ is condition evaluation of $\mathrm{i}^{\text {th }}$ element and $\mathrm{WF}_{\mathrm{i}}$ is the weight factor of $\mathrm{i}^{\text {th }}$ element. Based on the DI index, a bridge condition could be described with grades A to E (Table 4).

Table 4. Assessment of bridge condition in Korea.

\begin{tabular}{ccc}
\hline Rate & DI & Description \\
\hline A & $0 \leq \mathrm{DI}<0.13$ & Perfect \\
B & $0.13 \leq \mathrm{DI}<0.26$ & Minor problem in secondary elements \\
C & $0.26 \leq \mathrm{DI}<0.49$ & Minor problem in primary elements \\
D & $0.49 \leq \mathrm{DI}<0.79$ & Problem in primary elements \\
E & $0.79 \leq \mathrm{DI}$ & Serious problem in primary elements \\
\hline
\end{tabular}

In the United States, there are various approaches to assess the condition of the bridge. For example, the California Department of Transportation defines BHI based on Eq. (4). This index varies from 0 for the worst bridge condition to 100 for the healthiest bridge condition [22].

$$
\mathrm{BHI}=\left[\Sigma(\mathrm{CEV}) / \sum(\mathrm{TEV})\right] \times 100,
$$

where $\mathrm{BHI}$ is bridge health index, $\mathrm{CEV}$ is current element value and TEV is total element value.

CEV and TEV can be calculated according to the following equations [22]:

$$
\begin{gathered}
\mathrm{CEV}=\sum\left(\mathrm{QCS}_{\mathrm{i}} \times \mathrm{WF}_{\mathrm{i}}\right) \times \mathrm{FC}, \\
\mathrm{TEV}=\mathrm{TEQ} \times \mathrm{FC},
\end{gathered}
$$

where TEQ is total element quantity, FC is failure costs of element, $\mathrm{QCS}_{i}$ is quantity in condition state $i$, and WF is weight factor. 
In the United States, transportation departments report a set of data called national bridge inspection (NBI). Based on the physical condition of the bridge, the bridge is assigned score in the range of 0 to 9 [23]. The assessment procedure is presented in Table 5.

Table 5. Assessment of bridge condition based on NBI.

\begin{tabular}{|c|c|c|}
\hline Rate & State & Description \\
\hline 9 & Excellent & A new bridge \\
\hline 8 & Very good & No problems noted \\
\hline 7 & Good & Some minor problems \\
\hline 6 & Satisfactory & Structural elements show some minor deterioration \\
\hline 5 & Fair & $\begin{array}{l}\text { All primary structural elements are sound but may have minor section loss, deterioration, } \\
\text { spalling or scour }\end{array}$ \\
\hline 4 & Poor & Advanced section loss, deterioration, spalling, scour \\
\hline 3 & Serious & $\begin{array}{l}\text { Loss of section, etc., has affected primary structural components; Local failures are possible. } \\
\text { Fatigue cracks in steel or shear cracks in concrete may be present }\end{array}$ \\
\hline 2 & Critical & $\begin{array}{l}\text { Advanced deterioration of primary structural elements. Fatigue cracks in steel or shear cracks } \\
\text { in concrete may be present or scour may have removed structural support. Unless closely } \\
\text { monitored it may be necessary to close the bridge until corrective action is taken }\end{array}$ \\
\hline 1 & $\begin{array}{l}\text { Imminent } \\
\text { failure }\end{array}$ & $\begin{array}{l}\text { Major deterioration or loss of section in critical structural components or obvious vertical or } \\
\text { horizontal movement affecting structural stability. Bridge is closed to traffic but corrective } \\
\text { action may allow it to be returned to light service }\end{array}$ \\
\hline 0 & Failed & Out of service. Beyond corrective action \\
\hline
\end{tabular}

The US departments of transportation often uses a computer program to assess bridge conditions. This program is based on Eq. (7) [23].

$$
\mathrm{SR}=\mathrm{S}_{1}+\mathrm{S}_{2}+\mathrm{S}_{3}-\mathrm{S}_{4}
$$

where SR is Sufficiency rating, $S_{1}$ is the parameter related to structural safety, $S_{2}$ is the parameter related to bridge serviceability and functionality, $S_{3}$ is the parameter related to user requirements and $S_{4}$ is the parameter related to reductive coefficients based on structure type and traffic safety.

SR indicates the bridge sufficiency to remain in service, where SR has a maximum rating of $100 \%$, indicating complete bridge sufficiency, and a minimum rating of $0 \%$, indicating complete bridge deficiency. The parameters $S_{1}, S_{2}, S_{3}$ and $S_{4}$ have a weight importance of $55 \%, 30 \%, 15 \%$ and $13 \%$, respectively. FHWA uses SR to allocate rebuilding funds so that [23]:

- If $\mathrm{SR}<50$, the bridge is eligible for replacement;

- If $50<\mathrm{SR}<80$, the bridge is eligible for rehabilitation.

In Australia, Rashidi et al. presented an overall working framework for bridge infrastructure management. This framework had two phases, including project ranking and remediation planning. The engaged factors of this framework were weighting by expert judgments and employing AHP [24]. In phase project ranking, they presented a model for prioritizing based on priority index (PI). A bridge with higher PI takes priority for maintenance [25]:

$$
\mathrm{PI}=0.6(\mathrm{SE})+0.2(\mathrm{FE})+0.2(\mathrm{CIF})
$$

where PI is priority index, SE is structural efficiency index, FE is functional efficiency, and CIF is client impact factor. In phase remediation planning,

In phase remediation planning, the problem was modeled in a hierarchical order by a simplified hierarchical analysis process (S-AHP). This hierarchy consists of at least three main levels: goal, criteria, and alternatives. The goal is the remediation strategy. The criteria require to be broken down into more specific sub-criteria introduced as attributes in an extra level of the hierarchy. Each criterion has a weight indicating its importance. These weights are defined by the decision-makers. The final level is added for 
the remediation treatment alternatives. This procedure is flexible and can vary for different projects. Therefore, criteria, rehabilitation strategies, and even the number of levels can be different in various cases [26,27].

In Turkey, the following technique is used for the assessment of a bridge conditions and its elements [28]:

$$
\begin{gathered}
\mathrm{CR}(\mathrm{e})_{\text {element, } \mathrm{W}}=\sum \mathrm{j}\left(\left(\sum\left(\mathrm{WP} \mathrm{dt}, \mathrm{i}, \mathrm{j}_{\mathrm{j}} \times \mathrm{r}_{\mathrm{j}}\right)\right) /\left(\sum \sum\left(\mathrm{WP}_{\mathrm{dt}, \mathrm{i}, \mathrm{n}} \times \mathrm{r}_{\mathrm{n}}\right)\right)\right), \mathrm{j}=1, \ldots, \mathrm{S} ; \mathrm{i}=1, \ldots, \mathrm{d} ; \\
\mathrm{n}=1, \ldots, \mathrm{S} \\
\mathrm{CR}(\mathrm{b})_{\text {bridge }, \mathrm{W}}=\sum\left(\mathrm{W}_{\mathrm{e}} \times \mathrm{CR}(\mathrm{e})_{\text {element }, \mathrm{W})}\right) / 100, \mathrm{e}=1, \ldots, \mathrm{ne}_{\mathrm{e}}
\end{gathered}
$$

where $C R(e)$ element, $W$ is weighted assessment of conditions for element $\mathrm{e}, \mathrm{n}_{\mathrm{e}}$ is total number of bridge elements, $W P_{(d t, i, j)}$ is weighted percentages for damage type i under condition $j, s$ is total conditions, $\mathrm{d}$ is total number of damages, $\mathrm{r}_{j}$ is damage impact distribution coefficient, $C R(b)$ bridge, $W$ is weighted assessment of bridge $b$ and $W_{e}$ is weight importance of $r_{j}$ element. In Figure 2, the weight importance of elements for a conventional concrete bridge is shown.

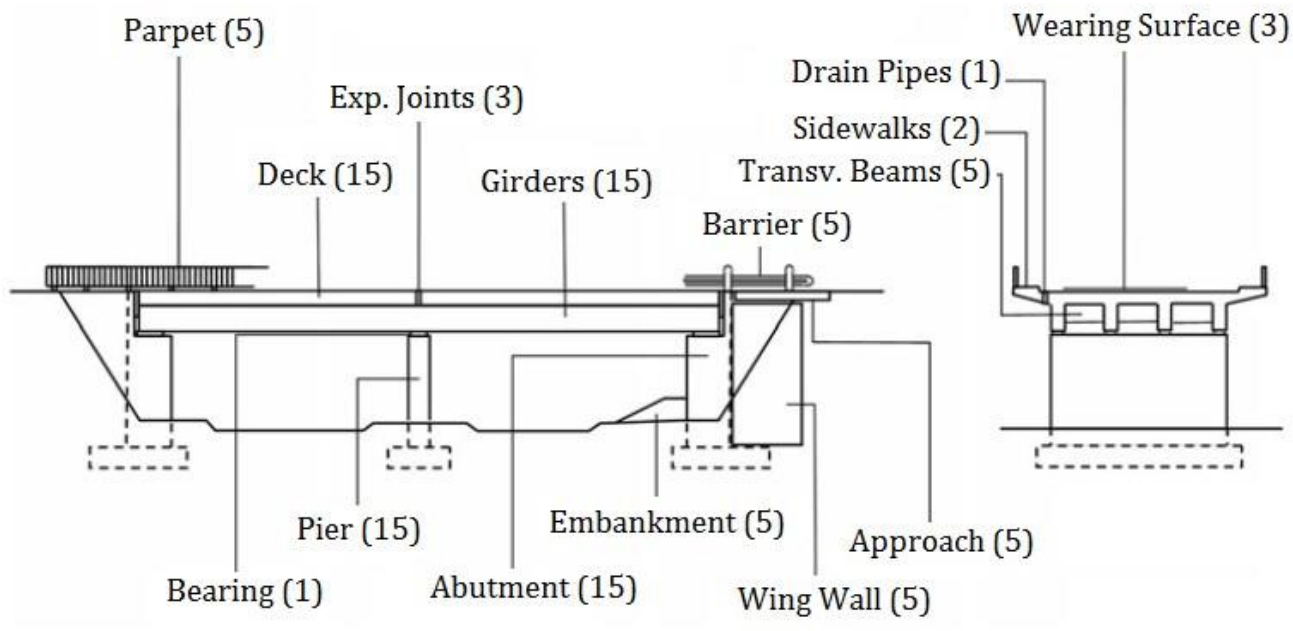

Figure 2. Weight importance of elements for a conventional concrete bridge.

In the first part of this section, an overview of the most important studies is relating to methods of assessing the condition of bridges in different countries is presented. After reviewing these works, the authors found that there are two main gaps in these methods. First, the majority of these methods take into account limiting factors in the assessment. In fact, none of the bridge assessment methods comprehensively take into account all the factors influencing the condition of bridges. Some of these methods are limited to the structural elements of the bridge and the factors affecting them. Second, some approaches tried to provide a more comprehensive model by considering other affecting factors, but they have presented new factors in general and only in one or two limited indicators. The authors believe that the factors should be examined in more detail than what is presented in these methods. To solve these two problems, the authors focus on a model that includes all the factors affecting the bridge condition. The method presented in this study involves crucial factors such as structural condition, drainage status, geotechnical and seismic factors, and safety. Indeed, the authors have considered other critical factors such as traffic loading, pavement, mechanical and electrical facilities, and strategic importance as separate indicators in the proposed method. Another gap that has been followed in this study is the lack of a comprehensive model for Iran. Therefore, the proposed method is based on the condition of bridges in Iran.

\section{Method}

\subsection{Bridge condition index (BCI)}


One of the major concerns of organizations in charge of bridges is that repairs and maintenance of bridges should be implemented with respect to financial constraints. The bridge maintenance is a costly and long-term project, which has led to the development of various scientific tools and methods for optimal budget allocation [29]. Before allocating any budget, it is necessary to determine the current condition of the bridge and its present and possibly future needs. The key to successful assessment of a bridge condition is to recognize various damages. Bridge damage is a slow, progressive and continuous process that is influenced by the imposing load, conditions of various bridge components, environmental factors and the properties of materials [17].

Many researchers have argued that the damage process is a blend of several mechanisms, such as corrosion, creep, shrinkage, cracking, fatigue, etc. [30]. The bridge damage is induced by a host of factors such as traffic, rainfall, freezing and melting cycles, climate change and pollution, which can eventually lead to bridge failure [17]. The bridge failure can be either structural or functional. The methods for assessing various components of a bridge and their relative significance are key concepts in BMS [31].

Different countries employ diverse methods to evaluate bridges so that they can develop a priority plan for bridge repair and maintenance with respect to budget constraints. Using a series of indicators is one of the most commonly used decision-making methods to prioritize maintenance. One perquisite of such indicators is determining the relative importance of different bridge components. Indicators can be categorized into two broad categories [17]:

1. Bridge Health Index (BHI)

2. Maintenance Priority Index (MPI)

The BHI is generally calculated as follows [17]:

$$
\mathrm{BHI}=\sum \mathrm{W}_{\mathrm{i}} \times \mathrm{C}_{\mathrm{i}}, \mathrm{i}=1, \ldots, \mathrm{n}
$$

where $\mathrm{BHI}$ is bridge health index, $W_{i}$ is the weight of $i^{\text {th }}$ element, $C_{i}$ is the condition of $i^{\text {th }}$ element and $\mathrm{n}$ is the number of bridge elements.

Moreover, MPI is usually calculated using the following equation [32]:

$$
\mathrm{MPI}=\sum \mathrm{K}_{\mathrm{i}} \times \mathrm{F}_{\mathrm{i}}(\mathrm{a}, \mathrm{b}, \mathrm{c}, \ldots),
$$

where MPI is maintenance priority index, $K_{i}$ is the weight of $i^{\text {th }}$ damage, $F_{i}$ is $i^{\text {th }}$ damage and $a, b, c$, etc. are damage characteristics.

The above indicators, BHI and MPI, aim to determine the condition of the in-service bridges. In fact, both these indicators are somehow the same as the BCI. In this study, a method for determining BCI is proposed that is structurally similar to BHI.

\subsection{Analytical hierarchy process (AHP)}

Introduced by Thomas Satty in 1980, the analytic hierarchy process (AHP) provides a mechanism for switching the criteria rating into weights [5,33]. In AHP, a pair-wise comparison of the relative importance of criteria results in criteria ranking. In fact, AHP is an effective and powerful tool for multi-criteria decision-making approaches. AHP is easy to apply and helps engineers obtain the final ranking from the individual evaluations, and finally, select an optimal alternative [34]. Because of these features, AHP has been used in bridge engineering in the past decades. Generally, the AHP can be included in the following steps [35]:

1. Constructing the pair-wise comparison judgment matrix.

2. Determining the weight of decision elements.

3. Controlling the compatibility index.

The purpose of this study is to present a bridge condition index (BCI) for evaluating and prioritizing MR\&R programs. For this purpose, first, eight indices affecting the quality of the bridge are introduced: structural index, hydrology and climate index, safety index, bridge performance index (load impact), geotechnical and seismic index, strategic importance index, facilities index, and traffic and pavement index. To determine $\mathrm{BCI}$, we need to evaluate the relative importance of these Indices. In this study, the rela- 
tive importance of indices is expressed in terms of weights, which are measured using the AHP method. AHP is an analytical method that allows making appropriate decisions by considering qualitative, quantitative, and mixed criteria. This is based on a dual comparison system that allows users to obtain indicators and select the target [36]. In cases where AHP is used for decision-making, first a proper hierarchy tree should be designed. This hierarchy tree has different levels depending on the type of problem under investigation. The first level necessarily indicates the goal of decision making, and the last level represents the alternatives that could be compared. Figure 3 shows the initial decision tree of this research.

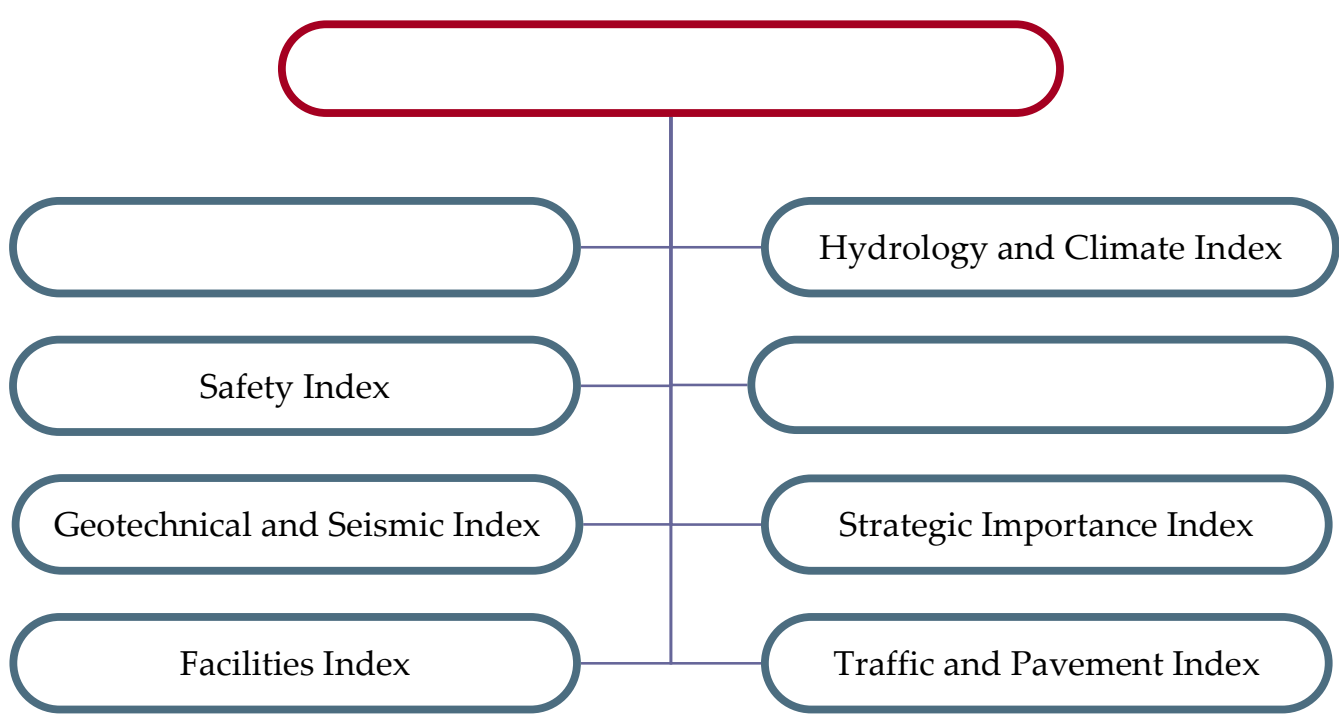

Figure 3. Initial decision tree in this study.

In the next step, a series of questionnaires about the relative importance of indices are designed and distributed among experts. The experts group participating in this study comprises of a number of specialists and advisors involved in maintenance and reinforcement of bridges as well as university professors with relevant expertise. In these questionnaires, which are in form of tables, experts are asked to assign a value between 0 to 10 based on their technical experience and expertise to dual comparisons in each row of the table. Table 6 shows the final results of this survey. Each table cell displays the relative importance of a row proportional to its corresponding column. In Table 6, if a row index outweighs a column index, a value between 1 and 10, and if the column index outweighs the row index, a value between 0 and 1 is assigned and value 1 is used at the diameter of table. It should also be noted that numbers in Table 6 represent the average views of experts. In next step, values shown in Table 6 are entered in Expert Choice software to calculate the relative weights of each index (see Table 7).

Table 6. Relative importance of indices based on a survey of experts.

\begin{tabular}{|c|c|c|c|c|c|c|c|c|}
\hline Index & 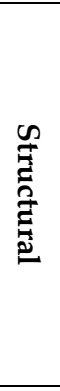 & 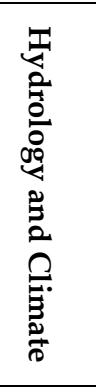 & $\begin{array}{l}\infty \\
\stackrel{0}{0} \\
\frac{\mathscr{D}}{4}\end{array}$ & 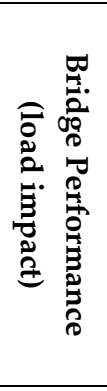 & 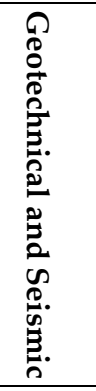 & 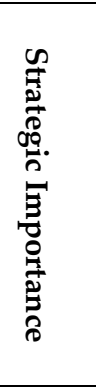 & 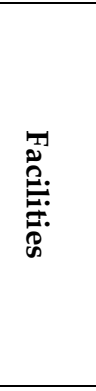 & 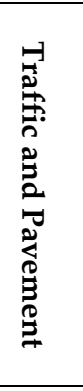 \\
\hline Structural & 1 & 5.271 & 3.152 & 4.581 & 1.877 & 3.13 & 6.075 & 3.578 \\
\hline Hydrology and Climate & & 1 & 1.037 & 1.382 & 0.788 & 1.377 & 1.871 & 0.941 \\
\hline Safety & & & 1 & 2.613 & 1.633 & 1.489 & 3.318 & 2.074 \\
\hline
\end{tabular}




\begin{tabular}{c|ccccc}
\hline Bridge Performance (load impact) & 1 & 0.761 & 1.164 & 2 & 1.154 \\
Geotechnical and Seismic & & 1 & 2.859 & 3.133 & 2.216 \\
Strategic Importance & & & 1 & 2.766 & 1.75 \\
Facilities & & & & 1 & 0.975 \\
Traffic and Pavement & & & & & 1 \\
\hline
\end{tabular}

Table 7. Relative weights of indices and compatibility rating

\begin{tabular}{|c|c|c|c|c|c|c|c|c|}
\hline Index & 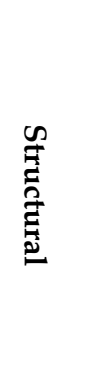 & 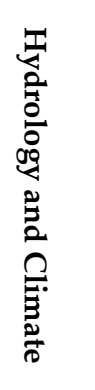 & $\underset{\substack{0 \\
\frac{0}{4}}}{\stackrel{\infty}{4}}$ & 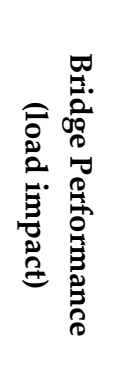 & 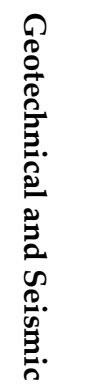 & 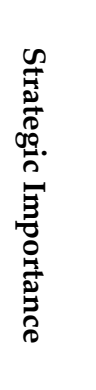 & 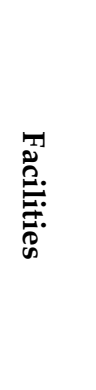 & 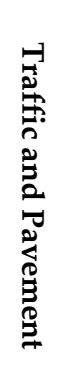 \\
\hline $\begin{array}{c}\text { Relative weight } \\
\text { compatibility rating }\end{array}$ & 0.331 & 0.097 & 0.146 & 0.080 & 0.143 & 0.088 & 0.046 & 0.068 \\
\hline
\end{tabular}

As indicated in Table 7, the relative weight of indices was calculated. This table contains additional information called compatibility rating. It is the mechanism that determines the adaptability of comparisons, indicating the extent to which the priorities selected by the group or the priorities of the mixed table are reliable. According to the experience, if the compatibility rate is less than 0.1 , the adaptability of comparisons is acceptable; otherwise, the comparisons need be repeated [35].

After determining the relative weight of indices, they are scored in terms of bridge conditions. First, each index is divided into a number of sub-indices. Figure 4 shows the completed decision tree in this study. Then, all damage types are defined for each sub-index. Finally, a series of questionnaires about damage scores are designed and distributed among the experts. They express their views about damage scores based on their experience and expertise. The scores collected in this step represent the average scores of all questionnaires. The numerical values of scores are in the range of 0 to 100 . This process is carried out for each sub-index, and the results are presented in the next subsections. With the exception of the structural index, sub-indices of each index are of the same importance. The structural index consists of four sub-indices (deck, girder, bent-abutment-wall, and foundation). To determine the relative weights of these sub-indices, a new questionnaire was designed and distributed among experts. By gathering the experts' views and analyzing them using Experts Choice software, the relative weights of mentioned sub-indices were calculated. 


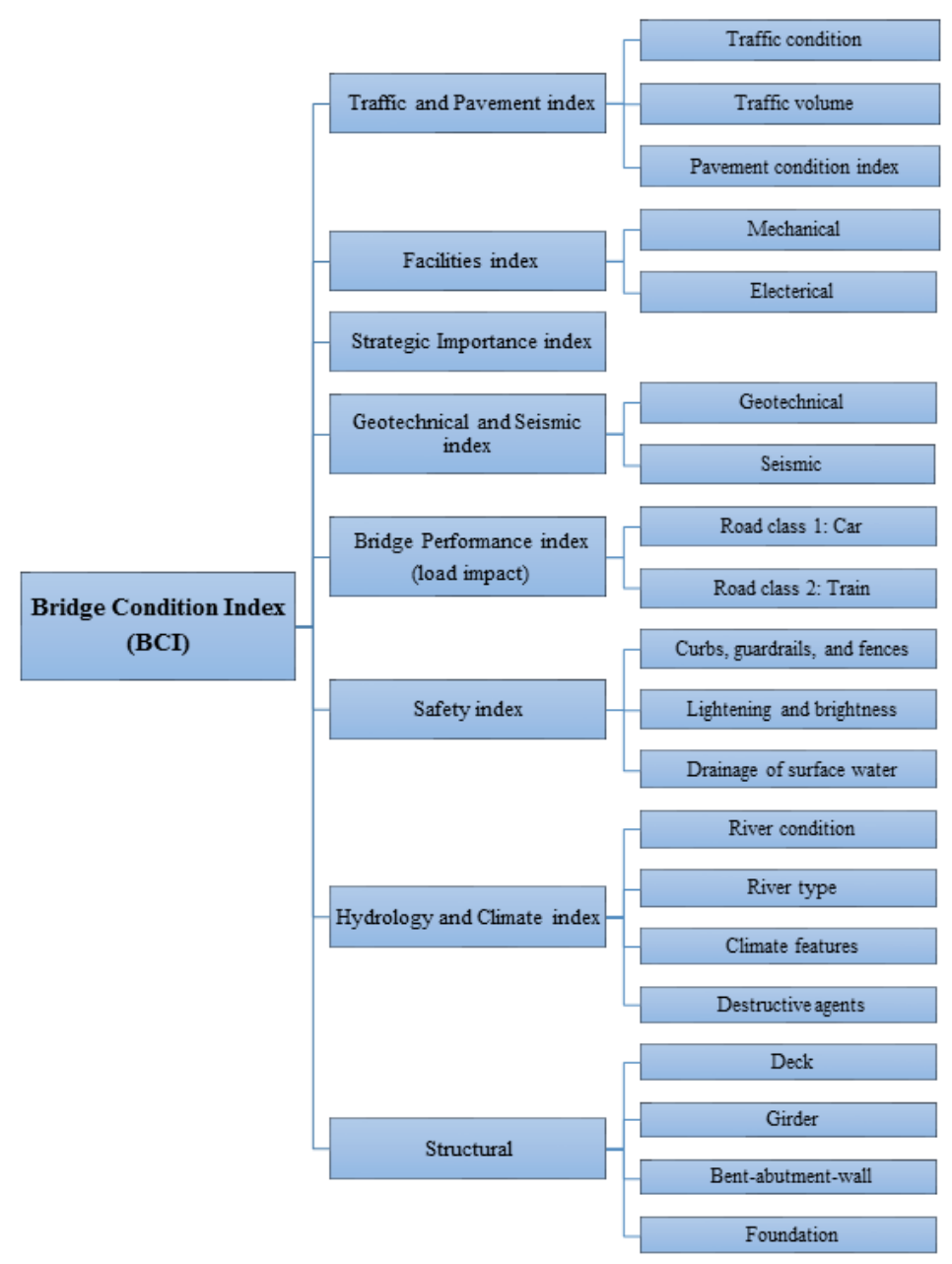

Figure 4. Final decision tree in this study.

Now, the BCI can be calculated. For each bridge, first, the score of each sub-index is determined based on the bridge inspection. After that, the score of each index is calculated based on their sub-indices score. BCI can be determined based on Eq. (13):

$$
\mathrm{BCI}=\sum\left(\mathrm{X}_{\mathrm{i}} \times \mathrm{W}_{\mathrm{i}}\right), \mathrm{i}=1, \ldots, 8
$$

where $\mathrm{BCI}$ is the bridge condition index, $\mathrm{X}_{\mathrm{i}}$ is the score of $\mathrm{i}^{\text {th }}$ index and $\mathrm{W}_{\mathrm{i}}$ is the weight of $\mathrm{i}^{\text {th }}$ index.

\subsection{Sub-indices of $B C I$}

\subsubsection{Structural index}

The structural index describes the bridge condition in terms of the damage to the structural elements. In other words, it evaluates the structural condition of the bridge. For structural analysis, a bridge is divided into four sub-indices: deck, girder, bent-abutment-wall, and foundation. It should be noted that the joints are classified under the deck sub-index and bearing and support in the sub-index of bent-abutment-wall. Similar to the eight main indices, first, the relative weights of these four sub-indices are determined. Tables 8 and 9 present the relative importance and relative weights of sub-indices, respectively. Next, these four sub-indices are assigned a score based on the extent and severity of their damage. Table 10 shows the assigned score of each sub-indices for different conditions. These scores are extracted from experts' recommendations.

Table 8. Relative importance of sub-indices in structural index based on a survey of experts.

Sub-index Deck Girder $\quad$ Bent-abutment-wall Foundation




\begin{tabular}{c|cccc}
\hline Deck & 1 & 1 & 0.84 & 1.476 \\
Girder & & 1 & 1.644 & 1.94 \\
Bent-abutment-wall & & & 1 & 3.204 \\
Foundation & & & & 1 \\
\hline
\end{tabular}

Table 9. Relative weight of sub-indices in structural index (Results of Expert Choice software).

\begin{tabular}{cccccc}
\hline Sub-index & Deck & Girder & Bent-abutment-wall & Foundation & $\begin{array}{c}\text { compatibility } \\
\text { rating }\end{array}$ \\
\hline $\begin{array}{c}\text { Relative } \\
\text { weight }\end{array}$ & 0.247 & 0.32 & 0.297 & 0.136 & 0.04 \\
\hline
\end{tabular}

Table 10. Scores of Structural index based on a survey of experts.

\begin{tabular}{ccccc}
\hline \multirow{2}{*}{ Damage intensity } & \multicolumn{4}{c}{ Score (in the range of 0-100) } \\
\cline { 2 - 5 } & Deck & Girder & Bent-abutment-wall & Foundation \\
\hline Low & 95 & 95 & 90 & 95 \\
Mediate & 70 & 65 & 60 & 75 \\
High & 30 & 30 & 25 & 35 \\
\hline
\end{tabular}

The structural index score is the sum of weighted scores of its four sub-indices. In the bridge maintenance program, usually, more attention was allocated to structural sub-indices, and they were included with more details in the assessment. In other indices, however, evaluations were more general.

\subsubsection{Hydrology and Climate Index}

One of the factors determining bridge condition is hydrology and environmental factors affecting the bridge. This index focuses on river conditions, river type, climatic features, and the concentration of destructive matters (such as sulfates) in the water, soil, and air. In hydrology and climate index, four scores are assigned to each bridge based on river conditions, river type, climatic features, and concentration of destructive matters. The average value of these scores, with identical weight, indicates the value of hydrology and climate index. These scores are shown in Table 11 (in the range of 0 to 100). It should be noted that if there is no river in the path under the bridge, the sub-indices of river conditions and destructive matters would be removed.

Table 11. Scores of hydrology and climate index based on a survey of experts.

\begin{tabular}{cccc}
\hline River conditions & \multicolumn{1}{c}{ River type } \\
\hline Description & Score & Type & Score \\
\hline $\begin{array}{c}\text { There is no erosion in the riverbed or the erosion is trivial. } \\
\text { The amount of sedimentation and debris is negligible }\end{array}$ & 98 & $\begin{array}{c}\text { Area under the bridge is } \\
\text { not a river path }\end{array}$ & 98
\end{tabular}

The riverbed has eroded slightly. There are signs of depositions in the upstream and downstream. Further analysis is required to detect failures

The erosion of the riverbed is critical and concerning.

There are enormous amounts of sedimentations around the bridge. Serious measures have to be taken.
58 There is seasonal river flowing under the bridge.

There is permanent river flowing under the bridge.

Table 11. Continued. 


\begin{tabular}{|c|c|c|c|}
\hline Description & Score & $\begin{array}{c}\text { Quality of protection } \\
\text { against destructive matters }\end{array}$ & Score \\
\hline $\begin{array}{l}\text { Mild (there are no invasive agents such as moisture, } \\
\text { transpiration, freezing and melting cycle, corrosive sub- } \\
\text { stances, etc.) }\end{array}$ & 93 & Very good & 95 \\
\hline $\begin{array}{l}\text { Medium (conditions that are occasionally exposed to } \\
\text { moisture and transpiration, and elements that are per- } \\
\text { manently exposed to non-invasive soils and water, or } \\
\text { underwater with a } \mathrm{pH}>5 \text { ) }\end{array}$ & 80 & Good & 76 \\
\hline $\begin{array}{l}\text { Severe (extreme humidity or transpiration, or freezing } \\
\text { and thawing cycle, elements immersed in water, such } \\
\text { that one surface is exposed to air, elements in chlorine ion } \\
\text { air, elements exposed to corrosion caused by the use of } \\
\text { anti-freezing agents) }\end{array}$ & 54 & Medium & 49 \\
\hline $\begin{array}{l}\text { Extremely severe (conditions that are exposed to gases, } \\
\text { water and static sewage with a pH of up to } 5 \text {, corrosive } \\
\text { matters, moisture with extreme icing and melting) }\end{array}$ & 35 & Bad & 14 \\
\hline $\begin{array}{l}\text { Exceptionally severe (conditions subject to extreme ero- } \\
\text { sion, flowing water and sewage with a maximum } \mathrm{pH} \text { of } \\
\text { 5) }\end{array}$ & 20 & & \\
\hline
\end{tabular}

\subsubsection{Safety index}

This index includes parameters that affect the safety of the bridge. There are several examples, including the beauty and proper serviceability of curbs, absence of any crack, fracture and delaminated curbs, proper functioning of the guardrails and fences, lighting and brightness and the efficiency of the drainage system to provide sufficient friction coefficient. The score of this index was calculated based on Table 12 . The safety rating score is the average score of the three sub-indices in Table 12.

Table 12. Safety index Scores based on a survey of experts (on a scale of 0-100).

\begin{tabular}{cccccc}
\hline $\begin{array}{c}\text { Curbs, guardrails and } \\
\text { fences }\end{array}$ & Lighting and brightness & & Drainage of surface water \\
\hline Description of defects & Score & Conditions & Score & Drainage condition & Score \\
\hline No repair is needed & 98 & $\begin{array}{c}\text { Trivial dazzling, excel- } \\
\text { lent color rendering, } \\
\text { broad sight }\end{array}$ & 94 & $\begin{array}{c}\text { Perfect drainage, ade- } \\
\text { quate friction coefficient }\end{array}$ & 96 \\
Partial repair is needed & 67 & $\begin{array}{c}\text { Slight dazzling, color } \\
\text { rendering and sight are } \\
\text { relatively desirable }\end{array}$ & 66 & $\begin{array}{c}\text { Drainage for securing } \\
\text { desirable friction }\end{array}$ \\
Major repair is required & 14 & $\begin{array}{c}\text { Extreme dazzling, low } \\
\text { color rendering and lim- } \\
\text { ited sight }\end{array}$ & 23 & $\begin{array}{c}\text { Improper drainage, unde- } \\
\text { sirable friction coefficient }\end{array}$ & 27 \\
\hline
\end{tabular}

\subsubsection{Load impact index}

In bridges that are under heavy loads or dynamic loads, damages are more common. The dynamic load imposed on a railway bridge is higher than that of a road bridge. Moreover, bridges that are often used for crossing heavy vehicles, such as a trailer or trucks, are more likely to be damaged than bridges used for light traffic. This is considered by the impact load index. Based on factors such as the use of bridge for vehicles or train, or the type of road it serves, the scores of this index are determined according to Table 13. The load impact score is the average score of the two sub-indices in Table 13. 
Table 13. Load impact index scores based on a survey of experts (on a scale of 0 to 100).

\begin{tabular}{ccc}
\hline Road class & Car & Train \\
\hline Freeway & 40 & 30 \\
Highway and major road & 45 & 40 \\
Minor road & 70 & 60 \\
Rural road & 85 & \\
Metro and monorail & & 70 \\
\hline
\end{tabular}

\subsubsection{Geotechnical and seismic index}

The quality of soil under the bridge foundation, seismicity of the region, and its geological structure affect the behavior of the bridge during an earthquake and its settlement, which consequently affect the bridge condition. This index is calculated based on Table 14. The score of the geotechnical and seismic index is obtained by averaging scores of geotechnical and seismic sub-indices.

Table 14. Geotechnical and seismic index scores based on a survey of experts (on a scale of 0 to 100).

\begin{tabular}{cccc}
\hline \multicolumn{2}{c}{ Geotechnical } & \multicolumn{2}{c}{ Seismic } \\
\hline Earth type & Score & Seismic area type & Score \\
\hline I & 92 & Low relative risk & 80 \\
II & 71 & Medium relative risk & 63 \\
III & 47 & High relative risk & 40 \\
IV & 26 & Very high relative risk & 23 \\
\hline
\end{tabular}

\subsubsection{Strategic importance index}

This index indicates the importance of the bridge location in terms of regional, strategic, and political considerations. The score of this index is computed according to Table 5. Strategic areas include hospitals (with more than 500 beds), military centers, crisis management centers, and fire stations.

Table 15. Scores of the strategic importance index based on a survey of experts (on a scale of 0 to 100).

\begin{tabular}{cc}
\hline The importance of strategic bridge & Score \\
\hline High importance (links two strategic areas) & 89 \\
Medium importance (links streets and non-strategic arterial) & 55 \\
Low importance (other bridges) & 29 \\
\hline
\end{tabular}

\subsubsection{Facilities Index}

This index is composed of two parts, including mechanical facilities and electrical facilities. The facilities index, which demonstrates the need for repairing electric or mechanical facilities of the bridge, is computed as the mean of scores of mechanical and electrical facilities, which can be deduced from Table 16.

Table 16. Scores of the facilities index based on a survey of experts (on a scale of 0 to 100).

\begin{tabular}{cccc}
\hline \multicolumn{2}{c}{ Mechanical facilities } & \multicolumn{2}{c}{ Electrical facilities } \\
\hline Drainage system & Score & Lighting condition & Score \\
\hline Fair & 97 & Good & 92 \\
Critical & 62 & Medium & 62 \\
Inappropriate & 30 & Unfair & 29 \\
\hline
\end{tabular}




\subsubsection{Traffic and pavement index}

One of the main parameters affecting bridge conditions is traffic and pavement. The score of the traffic sub-index is computed using Table 17. This sub-index includes two key factors: traffic condition and traffic volume. The average of scores of these two factors gives the score of traffic sub-index. We also apply the score of the pavement sub-index based on the pavement condition index (PCI). The value of PCI is equal to the pavement score. Finally, by averaging traffic and pavement scores, the score of traffic and pavement index is obtained.

Table 17. Scores of traffic sub-index based on a survey of experts (on a scale of 0 to 100).

\begin{tabular}{|c|c|c|c|}
\hline Traffic conditions & Score & Traffic volume & score \\
\hline $\begin{array}{l}\text { Very good (traffic facilities are perfectly working, full sight distance } \\
\text { and the number of lanes is standard) }\end{array}$ & 95 & Low & 89 \\
\hline $\begin{array}{l}\text { Good (traffic facilities are in relatively good condition, sight distance } \\
\text { is desirable in most areas and the number of lanes is appropriate) }\end{array}$ & 74 & medium & 68 \\
\hline $\begin{array}{c}\text { Moderate (Some of traffic facilities are in bad conditions and the } \\
\text { bridge has an undesirable curve) }\end{array}$ & 51 & Heavy & 51 \\
\hline $\begin{array}{l}\text { Bad (lanes are not enough, traffic facilities are not working, the } \\
\text { bridge has a horizontal and vertical curve together, the sight dis- } \\
\text { tance is not appropriate). }\end{array}$ & 12 & Very heavy & 26 \\
\hline
\end{tabular}

\section{Results and Discussion}

For implementing the proposed method, five bridges in Semnan province in Iran were selected. These case studies include the bridge of Shahmirzad road intersection, the bridge of Sari road intersection, the bridge on $73^{\text {rd }} \mathrm{km}$ of Semnan-Damghan road, the bridge on $6^{\text {th }} \mathrm{km}$ of Semnan-Jandaq road, and the bridge on $12^{\text {th }} \mathrm{km}$ of Semnan-Jandaq road. First, all bridges are inspected by the authors. After that, the BCI of all bridges was determined based on the proposed method in this study. The results of inspection and rating of bridges are given in the following subsections.

\subsection{The bridge of Shahmirzad road intersection}

The bridge of Shahmirzad road intersection is located in the city of Semnan, at the beginning of the Semnan-Shahmirzad Road. This bridge has two spans and acts as the overpass of the Mashhad-Tehran highway. The bridge is 45 meters in length and 23 meters in width and has three lanes in each direction. The BCI of this bridge is shown in Table 18.

Table 18. BCI calculation of Shahmirzad road intersection bridge

\begin{tabular}{lccccc}
\hline & Index & $\mathbf{W}_{\mathbf{i}}$ & $\mathbf{X}_{\mathbf{i}}$ & $\mathbf{W}_{\mathbf{i}} \times \mathbf{X}_{\mathbf{i}}$ & $\mathbf{B C I}=\sum\left(\mathbf{W}_{\mathbf{i}} \times \mathbf{X}_{\mathbf{i}}\right)$ \\
\hline $\mathbf{1}$ & Structural & 0.331 & 93.765 & 31.036 & \\
$\mathbf{2}$ & Hydrology and Climate & 0.097 & 95.5 & 9.264 & \\
$\mathbf{3}$ & Safety & 0.146 & 66 & 9.636 & \\
$\mathbf{4}$ & Bridge Performance (load impact) & 0.08 & 45 & 3.6 & 72.849 \\
$\mathbf{5}$ & Geotechnical and Seismic & 0.143 & 55.5 & 7.937 & \\
$\mathbf{6}$ & Strategic Importance & 0.088 & 55 & 4.84 & \\
$\mathbf{7}$ & Facilities & 0.046 & 46 & 2.116 & \\
$\mathbf{8}$ & Traffic and Pavement & 0.068 & 65 & 4.42 & \\
\hline
\end{tabular}

\subsection{The bridge of Sari road intersection}


This bridge is located in Damghan, at the intersection of Damghan Road and Damghan-Sari Road. The bridge has two spans and two lanes and is 25 meters in length and 9 meters in width. A summary of the BCI calculation of this bridge is presented in Table 19.

Table 19. BCI calculation of Sari road intersection bridge.

\begin{tabular}{lccccc}
\hline & Index & $\mathbf{W}_{\mathbf{i}}$ & $\mathbf{X}_{\mathbf{i}}$ & $\mathbf{W}_{\mathbf{i}} \times \mathbf{X}_{\mathbf{i}}$ & $\mathbf{B C I}=\sum \mathbf{W}_{\mathbf{i}} \times \mathbf{X}_{\mathbf{i}} \mathbf{)}$ \\
\hline $\mathbf{1}$ & Structural & 0.331 & 93.765 & 31.036 & \\
$\mathbf{2}$ & Hydrology and Climate & 0.097 & 95.5 & 9.264 & \\
$\mathbf{3}$ & Safety & 0.146 & 63 & 9.198 & \\
$\mathbf{4}$ & Bridge Performance (load impact) & 0.08 & 45 & 3.6 & 73.221 \\
$\mathbf{5}$ & Geotechnical and Seismic & 0.143 & 55.5 & 7.937 & \\
$\mathbf{6}$ & Strategic Importance & 0.088 & 55 & 4.84 & \\
$\mathbf{7}$ & Facilities & 0.046 & 30 & 1.38 & \\
$\mathbf{8}$ & Traffic and Pavement & 0.068 & 87.67 & 5.962 & \\
\hline
\end{tabular}

\subsection{Bridge on $73^{\text {rd }} \mathrm{km}$ of Semnan-Damghan road}

This bridge is located at $73^{\text {rd }} \mathrm{km}$ of Semnan-Damghan road. The bridge has five spans, a total length of 60 meters, a width of 10 meters, and two lanes. A summary of the BCI calculation of this bridge is depicted in Table 20.

Table 20. BCI calculation of the bridge on $73^{\text {rd }} \mathrm{km}$ of Semnan-Damghan road.

\begin{tabular}{|c|c|c|c|c|c|}
\hline & Index & $\mathbf{W}_{\mathrm{i}}$ & $X_{i}$ & $\mathbf{W}_{\mathrm{i}} \times \mathbf{X}_{\mathrm{i}}$ & $B C I=\sum\left(W_{i} \times X_{i}\right)$ \\
\hline 1 & Structural & 0.331 & 93.765 & 31.036 & \multirow{8}{*}{73.193} \\
\hline 2 & Hydrology and Climate & 0.097 & 66 & 6.402 & \\
\hline 3 & Safety & 0.146 & 72.33 & 10.56 & \\
\hline 4 & Bridge Performance (load impact) & 0.08 & 45 & 3.6 & \\
\hline 5 & Geotechnical and Seismic & 0.143 & 55.5 & 7.937 & \\
\hline 6 & Strategic Importance & 0.088 & 55 & 4.84 & \\
\hline 7 & Facilities & 0.046 & 62 & 2.852 & \\
\hline 8 & Traffic and Pavement & 0.068 & 87.67 & 5.962 & \\
\hline
\end{tabular}

\subsection{Bridge on $6^{\text {th }} \mathrm{km}$ of Semnan-Jandaq road}

This bridge is located at $6^{\text {th }} \mathrm{km}$ of Semnan-Jandaq road. It has one span with a length of 8 meters and a width of 7 meters in two lanes. Table 21 shows a summary of the BCI calculation of this bridge.

Table 21. BCI calculation of the bridge on $6^{\text {th }} \mathrm{km}$ of Semnan-Jandaq road.

\begin{tabular}{lccccc}
\hline & Index & $\mathbf{W}_{\mathbf{i}}$ & $\mathbf{X}_{\mathbf{i}}$ & $\mathbf{W}_{\mathbf{i}} \times \mathbf{X}_{\mathbf{i}}$ & $\mathbf{B C I}=\sum \mathbf{W}_{\mathbf{i}} \times \mathbf{X}_{\mathbf{i}} \mathbf{)}$ \\
\hline $\mathbf{1}$ & Structural & 0.331 & 86.355 & 28.583 & \\
$\mathbf{2}$ & Hydrology and Climate & 0.097 & 62.75 & 6.087 & \\
$\mathbf{3}$ & Safety & 0.146 & 52.67 & 7.69 & \\
$\mathbf{4}$ & Bridge Performance (load impact) & 0.08 & 70 & 5.6 & \multirow{2}{*}{62.172} \\
$\mathbf{5}$ & Geotechnical and Seismic & 0.143 & 43.5 & 6.22 & \\
$\mathbf{6}$ & Strategic Importance & 0.088 & 29 & 2.552 & \\
$\mathbf{7}$ & Facilities & 0.046 & 30 & 1.38 & \\
$\mathbf{8}$ & Traffic and Pavement & 0.068 & 45 & 3.06 & \\
\hline
\end{tabular}


This bridge is located at $12^{\text {th }} \mathrm{km}$ of Semnan-Jandaq road. The bridge has three spans with a total length of 20 meters and a width of 8 meters in two lanes. Table 22 shows the BCI of this bridge.

Table 22. BCI calculation of the bridge on $12^{\text {th }} \mathrm{km}$ of Semnan-Jandaq road.

\begin{tabular}{lccccc}
\hline & Index & $\mathbf{W}_{\mathbf{i}}$ & $\mathbf{X}_{\mathbf{i}}$ & $\mathbf{W}_{\mathbf{i}} \times \mathbf{X}_{\mathbf{i}}$ & $\mathbf{B C I}=\sum \mathbf{W}_{\mathbf{i}} \times \mathbf{X}_{\mathbf{i}} \mathbf{)}$ \\
\hline $\mathbf{1}$ & Structural & 0.331 & 83.635 & 27.683 & \\
$\mathbf{2}$ & Hydrology and Climate & 0.097 & 52.75 & 5.117 & \\
$\mathbf{3}$ & Safety & 0.146 & 35 & 5.11 & \\
$\mathbf{4}$ & Bridge Performance (load impact) & 0.08 & 70 & 5.6 & 56.768 \\
$\mathbf{5}$ & Geotechnical and Seismic & 0.143 & 43.5 & 6.22 & \\
$\mathbf{6}$ & Strategic Importance & 0.088 & 29 & 2.552 & \\
$\mathbf{7}$ & Facilities & 0.046 & 30 & 1.38 & \\
$\mathbf{8}$ & Traffic and Pavement & 0.068 & 45.67 & 3.106 & \\
\hline
\end{tabular}

According to Tables 18 to 22 , the bridge of $12^{\text {th }} \mathrm{km}$ of Semnan-Jandaq road has the lowest BCI among the five studied bridges $(\mathrm{BCI}=56.8)$. This bridge takes the highest priority for maintenance. The second priority is related to the bridge of $6^{\text {th }} \mathrm{km}$ of Semnan-Jandaq road with $\mathrm{BCI}=62.2$. The bridge at the beginning of Shahmirzad road, with $\mathrm{BCI}=72.8$, takes the third priority. Two other bridges with $\mathrm{BCI}=73.2$ take the lowest maintenance priority.

As mentioned in the earlier sections, a review of previous studies shows the lack of a comprehensive method for evaluating and prioritizing bridges. Each of the methods proposed by other researchers generally focuses on limited parts of the factors affecting the bridge conditions. In this study, the authors tried to include all the factors affecting the condition of bridges in their method. Examination of the results of five under-study bridges, with different characteristics, confirms that this method is feasible. Due to its simplicity, this method saves time and money in evaluating and prioritizing the bridge network. On the other hand, due to the high flexibility of this method, the methodology of the proposed method can be easily implemented anywhere in the world, and as a result, the calibration of the method is easily done. It is enough to design the relevant questionnaires, obtain the opinion of bridge engineering experts in each region, and easily inspect and prioritize bridges by determining scores and weights. This study helps remove another gap: There isn't an efficient and effective evaluation system for bridges in Iran.

\section{Conclusions}

This study presents a new method for the determination of BCI in concrete bridges. BCI constitutes eight indices and several sub-indexes. Each one of these indices and sub-indices has a specific score and importance weight. The scores and weights are assigned by experts of bridge engineering. After determining scores and weights, inspectors survey the bridge and assign a score to all sub-index based on their condition. Then, the score of each index is obtained. Finally, by summing the weighted scores of indices, BCI will be determined. The necessity of this research could be justified in the absence of any comprehensive and effective system or index for assessing the bridge conditions, especially in Iran. Due to financial constraints and the lack of qualified specialists, it is also crucial to provide solutions to overcome these shortcomings. Therefore, in this research, attempts were made to provide a new and simple method for assessing bridge conditions in order to optimize the management activities. The novelty of this study is in the scoring system because the scoring system is constructed by native experts' views. On the other hand, because of lacking a comprehensive and proper index in Iran, this paper is considered innovative. To test the proposed method, five bridges in Semnan province were inspected and their BCI was determined to prioritize bridges. 
Simplicity is one of the characteristics of the method proposed because it does not require the application of non-destructive equipment or laboratory tests, and it allows measuring BCI and prioritizing bridges for maintenance based on the visual evaluation of the damages and general characteristics of the bridge and its performance. Therefore, time and budget can be saved in this method. On the other hand, the experts' views, scores, and coefficients of relative importance may vary in different organizations or countries. Thus, the calibration of this method is only done by designing the questionnaire and collecting experts' views.

For future studies, this method can be applied or extended to the steel and stone (old) bridges to embrace all bridges. Also, by increasing the views of more experts, this method can be more comprehensive and efficient.

Author Contributions: Conceptualization, S.D. and H.G.T.; methodology, H.G.T. and N.K.; software, S.D. and N.K.; validation, H.G.T., N.K. and A.M.; formal analysis, S.D. and H.G.T.; investigation, N.K. and A.M.; resources, S.D.; data curation, H.G.T. and N.K.; writing-original draft preparation, S.D. and H.G.T.; writing - review and editing, N.K.; visualization, A.M.; supervision, H.G.T. and A.M.; project administration, H.G.T.; funding acquisition, A.M. All authors have read and agreed to the published version of the manuscript.

Funding: Please add: "This research received no external funding" or "This research was funded by NAME OF FUNDER, grant number XXX" and "The APC was funded by XXX". Check carefully that the details given are accurate and use the standard spelling of funding agency names at https://search.crossref.org/funding. Any errors may affect your future funding.

Conflicts of Interest: The authors declare no conflict of interest.

\section{References}

1. Liu, H.; Wang, X.; Tan, G.; He, X. System Reliability Evaluation of a Bridge Structure Based on Multivariate Copulas and the AHP-EW Method That Considers Multiple Failure Criteria. Applied Sciences 2020, 10, 1399.

2. Jeong, Y.; Kim, W.; Lee, I.; Lee, J. Bridge inspection practices and bridge management programs in China, Japan, Korea, and US. Journal of Structural Integrity and Maintenance 2018, 3, 126-135.

3. Patel, D.; Lad, V.; Chauhan, K.; Patel, K. Development of Bridge Resilience Index Using Multicriteria Decision-Making Techniques. Journal of Bridge Engineering 2020, 25, 04020090.

4. Karballaeezadeh, N.; Ghasemzadeh Tehrani, H.; Mohammadzadeh Shadmehri, D.; Shamshirband, S. Estimation of flexible pavement structural capacity using machine learning techniques. Frontiers of Structural and Civil Engineering 2020, 14, 1083-1096, doi:10.1007/s11709-020-0654-z.

5. Liu, H.; Wang, X.; Tan, G.; He, X.; Luo, G. System reliability evaluation of prefabricated RC hollow slab Bridges considering hinge joint damage based on modified AHP. Applied Sciences 2019, 9, 4841.

6. Karballaeezadeh, N.; Mohammadzadeh S, D.; Moazemi, D.; S Band, S.; Mosavi, A.; Reuter, U. Smart Structural Health Monitoring of Flexible Pavements Using Machine Learning Methods. Coatings 2020, 10, 1100, doi:10.3390/coatings10111100.

7. Akgul, F. Inspection and evaluation of a network of concrete bridges based on multiple NDT techniques. Structure and Infrastructure Engineering 2020, 1-20.

8. Ryall, M. Bridge management; CRC Press: 2009.

9. Wu, C.; Wu, P.; Wang, J.; Jiang, R.; Chen, M.; Wang, X. Critical review of data-driven decision-making in bridge operation and maintenance. Structure and Infrastructure Engineering 2020, 1-24.

10. Zhang, L.; Qiu, G.; Chen, Z. Structural health monitoring methods of cables in cable-stayed bridge: A review. Measurement 2021, 168, 108343.

11. Jeong, E.; Seo, J.; Wacker, J. Literature Review and Technical Survey on Bridge Inspection Using Unmanned Aerial Vehicles. Journal of Performance of Constructed Facilities 2020, 34, 04020113. 
12. Nili, M.H.; Taghaddos, H.; Zahraie, B. Integrating discrete event simulation and genetic algorithm optimization for bridge maintenance planning. Automation in Construction 2021, 122, 103513.

13. Moodi, F. Development of a Knowledge Based Expert System for the Repair and Maintenance of Concrete Structures. PhD. Thesis, Newcastle upon Tyne University, Newcastle upon Tyne, UK, 2001.

14. Rashidi, M.; Gibson, P. A methodology for bridge condition evaluation. 2012.

15. Wu, H.-C. A multi-objective decision support model for maintenance and repair strategies in bridge networks; Columbia University: 2008.

16. Abu Dabous, S.; Alkass, S. Decision support method for multi-criteria selection of bridge rehabilitation strategy. Construction Management and Economics 2008, 26, 883-893.

17. Wakchaure, S.S.; Jha, K.N. Determination of bridge health index using analytical hierarchy process. Construction Management and Economics 2012, 30, 133-149.

18. MOT. Standards for technical condition evaluation of highway bridges-JTG/T H21-2011. The Ministry of Transportation: Beijing, China, 2011.

19. MHURD. Technical code of maintenance for city bridge (CJJ99-2003). Beijing, China: Ministry of Housing and Urban-Rural Development, 2003.

20. MLIT. Manual for Bridge Periodic Inspection. Tokyo, Japan: Ministry of Land, Infrastructure, Transportation, and Tourism, 2014.

21. MOLIT. Guideline of safety inspection and in-depth safety inspection for structures. Sejong, South Korea: Ministry of Land, Infrastructure, and Transport, 2012.

22. Shepard, R.W.; Johnson, M.B. California bridge health index: A diagnostic tool to maximize bridge longevity, investment. TR News 2001.

23. FHWA. Bridge inspector's reference manual. FHWA NHI 12-049 2012.

24. Rashidi, M.; Gibson, P.; Ho, T.K. A New Approach to Bridge Infrastructure Management. 2013.

25. Rashidi, M.; Samali, B.; Sharafi, P. A new model for bridge management: Part A: condition assessment and priority ranking of bridges. Australian Journal of Civil Engineering 2016, 14, 35-45.

26. Rashidi, M.; Samali, B.; Sharafi, P. A new model for bridge management: Part B: decision support system for remediation planning. Australian Journal of Civil Engineering 2016, 14, 46-53.

27. Rashidi, M.; Lemass, B.P. A decision support methodology for remediation planning of concrete bridges. 2011.

28. Akgul, F. Bridge management in Turkey: a BMS design with customised functionalities. Structure and Infrastructure Engineering 2016, 12, 647-666.

29. Rogulj, K.; Kilić Pamuković, J.; Jajac, N. Knowledge-Based Fuzzy Expert System to the Condition Assessment of Historic Road Bridges. Applied Sciences 2021, 11, 1021.

30. Agrawal, A.K.; Kawaguchi, A.; Chen, Z. Deterioration rates of typical bridge elements in New York. Journal of Bridge Engineering 2010, 15, 419-429.

31. Hsu, H.; Chang, W.; Wang, R.; Cho, C.; Jiang, D. Small and medium size bridge maintenance sequence analysis by optimization technique. In Advances in Bridge Maintenance, Safety Management, and Life-Cycle Performance, Set of Book $\mathcal{E}$ CD-ROM, CRC Press: 2015; pp. 139-140.

32. Hearn, G. Condition data and bridge management systems. Structural engineering international 1998, 8, $221-225$.

33. Ikpong, A.; Chandra, A.; Bagchi, A. Alternative to AHP Approach to Criteria Weight Estimation in Highway Bridge Management. Canadian Journal of Civil Engineering 2020.

34. Phung, X.L.; Truong, H.S.; Bui, N.T. Expert system based on integrated fuzzy AHP for automatic cutting tool selection. Applied Sciences 2019, 9, 4308. 
35. Chen, L.; Deng, X. A modified method for evaluating sustainable transport solutions based on AHP and Dempster-Shafer evidence theory. Applied Sciences 2018, 8, 563.

36. Kuzman, M.K.; Grošelj, P.; Ayrilmis, N.; Zbašnik-Senegačnik, M. Comparison of passive house construction types using analytic hierarchy process. Energy and Buildings 2013, 64, 258-263. 